\title{
Controlled-release levodopa methyl ester/ benserazide-loaded nanoparticles ameliorate levodopa-induced dyskinesia in rats
}

This article was published in the following Dove Press journal:

International Journal of Nanomedicine

18 April 2012

Number of times this article has been viewed

\section{Xinxin Yang ${ }^{*}$ \\ Ruiyuan Zheng ${ }^{2 *}$ \\ Yunpeng $\mathrm{Cai}^{2}$ \\ Meiling Liao ${ }^{2}$ \\ Weien Yuan ${ }^{1,2}$ \\ Zhenguo Liu'}

'Department of Neurology, Xinhua Hospital (affiliated to Shanghai Jiaotong University School of Medicine), ${ }^{2}$ School of Pharmacy, Shanghai Jiaotong University, Shanghai, People's Republic of China

*Xinxin Yang and Ruiyuan Zheng contributed equally to this work
Correspondence: Zhenguo Liu

Department of Neurology, Xinhua Hospital (affiliated to Shanghai jiaotong University School of Medicine), 1665 Kongjiang Road, Shanghai 200092,

People's Republic of China

$\mathrm{Tel} / \mathrm{fax}+862165790000$

Email zhenguoliu2004@yahoo.com.cn

Weien Yuan

School of Pharmacy, Shanghai Jiao Tong University, 800 Dongchuan Road, Shanghai 200240, People's Republic of China

$\mathrm{Tel} / \mathrm{fax}+86$ 2I 34205072

Email yuanweien@I26.com
Background: Levodopa remains the most effective drug in the treatment of Parkinson's disease. However, long-term administration of levodopa induces motor complications, such as levodopa-induced dyskinesia. The mechanisms underlying levodopa-induced dyskinesia are not fully understood.

Methods: In this study, we prepared levodopa methyl ester (LDME)/benserazide-loaded nanoparticles, which can release LDME and benserazide in a sustained manner. Dyskinesia was induced in rats by repeated administration of levodopa then treated with LDME plus benserazide or the same dose of LDME/benserazide-loaded nanoparticles. Apomorphine-induced rotations and abnormal involuntary movements (AIMs) were measured on treatment days $1,5,10,15$, and 20 . In addition, the levels of phosphorylated dopamine- and cyclic adenosine monophosphate-regulated phosphoprotein of $32 \mathrm{kDa}$, extracellular signal-regulated kinases $1 / 2$, and $\Delta$ fosB were determined by Western blot. Tau levels were determined by Western blot and immunohistochemistry. Dynorphin levels in the striatum and cortex of rats were measured using enzyme-linked immunosorbent assay.

Results: Over the course of levodopa treatment, the rats developed abnormal AIMs, classified as locomotive, axial, orolingual, and forelimb dyskinesia. The degree of reduction of apomorphine-induced rotations was comparable in dyskinetic rats treated with LDME plus benserazide or LDME/benserazide-loaded nanoparticles. The axial, limb, and orolingual (ALO) AIMs of dyskinetic rats treated with LDME/benserazide-loaded nanoparticles were $14 \pm 2.5$, $9 \pm 2.0$, and $10 \pm 2.1$ on treatment days 10,15 , and 20 , respectively, which were significantly reduced compared with dyskinetic rats treated with LDME plus benserazide $(25 \pm 3.7,27 \pm 3.8$, and $25 \pm 3.5$, respectively). The locomotive AIMs of dyskinetic rats treated with LDME/ benserazide-loaded nanoparticles were $2.3 \pm 0.42,1.7 \pm 0.35$, and $1.6 \pm 0.37$ on treatment days 10,15 , and 20, respectively, which were also reduced compared with dyskinetic rats treated with LDME plus benserazide $(4.4 \pm 0.85,4.7 \pm 0.95$ and $4.8 \pm 0.37$, respectively). Western blot showed that the levels of phosphorylated dopamine- and cyclic adenosine monophosphateregulated phosphoprotein of $32 \mathrm{kDa}$, extracellular signal-regulated kinases $1 / 2$, tau, and $\Delta$ fosB in dyskinetic rats treated with LDME/benserazide-loaded nanoparticles were $134.6 \pm 14.1$, $174.9 \pm 15.1,134.2 \pm 19.3$, and $320.5 \pm 32.8$, respectively, which were significantly reduced compared with those of dyskinetic rats treated with LDME plus benserazide $(210.3 \pm 19.7$, $320.8 \pm 21.9,340.4 \pm 27.1$, and $620.7 \pm 48.3$, respectively). Immunohistochemistry indicated that the level of phosphorylated tau was $(7.2 \pm 1.1) \times 10^{4}$ in dyskinetic rats treated with LDME/ benserazide-loaded nanoparticles. However, the tau level was only $(14.6 \pm 2.3) \times 10^{4}$ in LDME plus benserazide-treated dyskinetic rats. There was a significant difference between the two groups. Enzyme-linked immunosorbent assay showed that dynorphin levels in the striatum and cortex of dyskinetic rats treated with LDME/benserazide-loaded nanoparticles were $5.7 \pm 1.2$ 
and $4.8 \pm 0.87$, respectively, which were significantly reduced compared with LDME plus benserazide-treated dyskinetic rats $(13.3 \pm 2.1$ and $8.1 \pm 1.1$ for the striatum and cortex, respectively).

Conclusion: Results suggest that LDME/benserazide-loaded nanoparticles can be used to reduce the expression of dyskinesia in dyskinetic rats.

Keywords: Parkinson's disease, levodopa-induced dyskinesia, $\Delta$ fosB, dopamine, cAMP-regulated phosphoprotein of $32 \mathrm{kDa}$, extracellular signal-regulated kinases $1 / 2$, tau

\section{Introduction}

Parkinson's disease (PD) is a progressive neurodegenerative disorder often observed in older patients. Levodopa has been used to treat the disease for many years. Although levodopa is effective for treating the motor disturbance associated with Parkinson's disease, ${ }^{1}$ long-term levodopa therapy is known to cause levodopa-induced dyskinesia (LID). ${ }^{2,3}$ The pathology of LID is poorly understood and, once established, it is difficult to treat. Numerous studies have shown that continuous dopamine stimulation can be used to reduce the expression of LID. ${ }^{4-6}$ Continuous dopamine stimulation can ameliorate the neuron adaptations in the striata of 6-hydroxydopamine (6-OHDA)-lesioned rats. We recently reported that levodopa methyl ester (LDME)/benserazide-loaded nanoparticles can be used to treat PD motor symptoms and reduce the expression of LID in a rat model of PD. ${ }^{7}$ However, whether LDME/ benserazide-loaded nanoparticles can be used to ameliorate established levodopa-induced dyskinesia in rats is not well understood. In this study, dyskinesia was induced in rats by repeated administration of levodopa. Then, the effects of LDME/benserazide-loaded nanoparticles on abnormal involuntary movements (AIMs) and the levels of phosphorylated dopamine- and cyclic adenosine monophosphate-regulated phosphoprotein of $32 \mathrm{kDa}$ (p-DARPP-32) at threonine 34, phosphorylated extracellular signal-regulated kinases $1 / 2$ (p-ERK1/2), $\Delta$ fosB, and dynorphin, which are closely associated with LID in rats, were investigated. Moreover, a recent study has shown that phosphorylated tau (p-tau) might be associated with LID. ${ }^{8}$ However, it remains unknown whether continuous dopamine stimulation can affect the phosphorylation of tau in PD rats. To this end, whether LDME/ benserazide-loaded nanoparticles can reduce the expression of p-tau in dyskinetic rats was also investigated.

\section{Materials and methods Materials}

LDME and benserazide hydrochloride were purchased from Sigma (St Louis, MO). Polyvinyl alcohol, dichloromethane (DCM), phosphoric acid, potassium dihydrogen phosphate, sodium phosphate dibasic anhydrous, and sodium chloride $(\mathrm{NaCl})$ were obtained from the Chinese Medicine Group
Chemical Reagent Corporation (Shanghai, People's Republic of China). Citric acid was supplied by Ning Pharmaceutical Co, Ltd (Taishan City, People's Republic of China). Methanol was purchased from Jiangsu Yonghua Fine Chemical Products Co, Ltd (Jiangsu, People's Republic of China). Poly(lactic-co-glycolic acid) 50/50 3A (47 KDa) and polylactic acid $(83 \mathrm{KDa})$ were obtained from Lakeshore Biopolymers Inc (Cincinnati, OH). Water was obtained from a Milli-Q purification system (Millipore, Molsheim, France).

\section{Preparation of LDME/benserazide-loaded nanoparticles}

We prepared LDME/benserazide-loaded nanoparticles as previously reported. ${ }^{7}$ Benserazide-loaded nanoparticles were prepared according to the water-in-oil-in-water emulsion solvent evaporation method. Benserazide was directly added to the DCM solution of poly(lactic-co-glycolic acid) $50 / 50$ $3 \mathrm{~A}(47 \mathrm{kDa}, 5 \% \mathrm{wt} / \mathrm{wt})$ and polylactic acid $(83 \mathrm{kDa}, 7.5 \%$ $\mathrm{wt} / \mathrm{wt})$. The rest of the procedure was the same as the method previously reported. ${ }^{7}$ If the salt or the organic phase was not removed completely, nanoparticles would gather together and the material would be difficult to inject. When the nanoparticles were initially formed by fast stirring, care had to be taken not to let the nanoparticles be exposed to the air. If this occurred, the nanoparticles would be destroyed when the dichloromethane volatilized quickly. Further, the process of microencapsulation was undertaken in dark conditions, to avoid light decomposition of the drugs.

\section{Morphological characteristics of the nanoparticles}

Microsphere shape and surface morphology were estimated using scanning electron microscopy. Nanoparticles were attached onto a metal stub using double-sided adhesive and exposed to gold spray under argon atmosphere for 10 minutes. Scanning electron microscopy images were taken using an FEI Sirion 200 (Hillsboro, OR) at a sputtering energy of $5 \mathrm{keV}$.

\section{In vitro release study}

Twenty milligrams of nanoparticles were accurately weighed then dispersed in $1 \mathrm{~mL}$ of dissolution medium 
(0.5835 $\mathrm{g} \mathrm{Na}_{2} \mathrm{HPO}_{4}$ and $1.5264 \mathrm{~g}$ citrate acid per $100 \mathrm{~mL}$ of distilled water, approximate $\mathrm{pH}$ of 3.5). The solution was rotated in a constant-temperature rotating basket (HZ-8812S, Taichang Scientific Education Apparatus Factory, Jiangsu, People's Republic of China; $37^{\circ} \mathrm{C}, 50 \mathrm{rpm}$ ). Samples were withdrawn at specified time intervals for analysis and replaced with equivalent volumes of fresh dissolution medium. LDME and benserazide concentrations were measured using a highperformance liquid chromatograph (WondaSil C18-WR, Shimadzu Corp, Kyoto, Japan).

LDME was analyzed at $280 \mathrm{~nm}$; the mobile phase consisted of water:methanol $=80: 20(\mathrm{pH}$ was adjusted to 3.5 using phosphoric acid). Benserazide was analyzed at $220 \mathrm{~nm}$; the mobile phase consisted of trichloroacetic acid:methanol:water $=1: 20: 100$. The concentrations of the samples were calculated using standard curves made in advance.

\section{Determination of encapsulation efficiency} After 20 days of in vitro release, the drug in the dissolution medium could not be detected by high-performance liquid chromatography (HPLC). Then the nanoparticles (20 mg) were dissolved in $0.5 \mathrm{~mL}$ of DCM in a vortex oscillator and the residual drug in the DCM solution was extracted three times using $0.5 \mathrm{~mL}$ dissolution medium. The residual drug was also examined by HPLC under the same conditions mentioned. The release amount in vitro and the residual drug amount were summed to calculate the total drug amount loaded in the nanoparticles. Encapsulation efficiency was calculated according to the following formula:

$$
\begin{aligned}
& \text { Encapsulation efficiency } \\
& \begin{array}{l}
=\frac{\text { Weight of encapsulated drug }}{\text { Weight of the total amount of drug used for encapsulation }} \\
\quad \times 100 \%
\end{array}
\end{aligned}
$$

\section{In vivo release study}

After analyzing the results of in vitro release, the formulation with the longest release time and an acceptable initial burst was chosen for the in vivo study. Following this, four healthy male rats each weighing approximately $500 \mathrm{~g}$ were subcutaneously injected with weighed nanoparticles dispersed in a liquid of suitable viscosity (60 mg mannitol and $50 \mathrm{mg}$ sodium carboxymethylcellulose per $10 \mathrm{~mL}$ of injectable water, $1 \mathrm{~g}$ nanoparticles dispersed in approximately $30 \mathrm{~mL}$ liquid). The amount of nanoparticles injected is given $500 \mathrm{mg}$.
Blood samples were collected from rats' tails at specified time intervals then centrifuged (5000 rpm, 5 minutes). Afterward, plasma proteins were removed by adding methanol (chromatographically pure) and centrifuging again (10,000 rpm, 20 minutes). Analysis of the samples was also carried out using HPLC under the same conditions previously mentioned. The concentration of samples was calculated by standard curves generated from drugs dissolved in methanol (chromatographically pure).

All protocols involving animals were approved by the Institutional Review Board of Xinhua Hospital and were performed according to the guidelines of the National Institutes of Health for the care and use of laboratory animals (NIH publication No. 80-23).

\section{6-hydroxydopamine lesions}

All animals received injections totaling $8 \mu \mathrm{g}$ 6-OHDA (Sigma, St Louis, MO). Coordinates were calculated using the rat brain atlas as follows: anterior-posterior: $-4.4 \mathrm{~mm}$, medial-lateral: $-1.2 \mathrm{~mm}$, dorsal-ventral: $-7.8 \mathrm{~mm} .{ }^{9}$ The tooth bar was set to $-2.4 \mathrm{~mm}$. One week after the injections, rats that exhibited stable apomorphine-induced rotational asymmetry of at least seven full turns per minute away from the lesioned side were selected for the following experiment.

\section{Treatment}

All valid PD rats were administrated with levodopa to induce expression of LID. Then all dyskinetic rats (AIMs score $>8$ ) were divided into two groups: levodopa + levodopa $(n=14)$ and levodopa + nanoparticle $(n=14)$. Rats in the levodopa + levodopa group were treated with LDME $(6 \mathrm{mg} / \mathrm{kg}$, subcutaneously) plus benserazide (15 mg/kg, subcutaneously) daily for 3 weeks. With the assumption that LDME/ benserazide-loaded biodegradable nanoparticles would gradually release the drug over at least 1 week, rats in the levodopa + nanoparticle group were treated with subcutaneous LDME/benserazide-loaded nanoparticles containing LDME (6 mg/kg, subcutaneously) plus benserazide (15 mg/kg, subcutaneously) once per week for 3 weeks. In addition, rats in the PD group $(\mathrm{n}=14)$ and sham group ( $n=14$, given sham-6-OHDA injections without expressing PD symptoms) were treated with a subcutaneous vehicle daily for 3 weeks.

\section{Apomorphine-induced rotations}

On test days, after administration of apomorphine $(0.5 \mathrm{mg} / \mathrm{kg}$, intraperitoneally), apomorphine-induced rotations were measured for 30 minutes. 


\section{Measurement of abnormal involuntary movements}

Rats were monitored for AIMs using a procedure similar to that described previously. ${ }^{10}$ On test days, rats were individually placed in plastic trays 5 minutes before drug treatment. Following injections, each rat was assessed for exhibition of ALO and locomotive movements. At 20-minute intervals (ie, 20, 40, 60, 80 minutes, etc), AIMs were rated for 60 seconds for each rat for a total of 2 hours, during which a severity score between 0 and 4 was assigned for each AIMs category. For each AIMs category, the scores for each time point were summed.

\section{Western blot}

Striatal tissues were homogenized $(1: 10 \mathrm{wt} / \mathrm{vol})$ in $20 \mathrm{mM}$ tris(hydroxymethyl)aminomethane hydrochloride ( $\mathrm{pH}$ 7.4), containing $1 \mathrm{mM} \mathrm{NaF}, 150 \mathrm{mM} \mathrm{NaCl}, 1 \%$ Triton $^{\mathrm{TM}} \mathrm{X}-100$ (Sigma-Aldrich, St Louis, MO), fresh protease inhibitor cocktail (CalBiochem, La Jolla, CA), and $100 \mu \mathrm{M}$ phenylmethylsulfonyl fluoride. Cytosols were prepared by centrifugation at $12,000 \mathrm{~g}$ for 10 minutes at $4^{\circ} \mathrm{C}$. Samples containing $40 \mu \mathrm{g}$ protein were separated on a $10 \%$ sodium dodecyl sulphate-polyacrylamide gel and electrophoretically transferred to polyvinylidene fluoride membranes (Bio-Rad, Hercules, CA) in tris-glycine transfer buffer. Membranes were then blocked in instant nonfat dried milk (5\% wt/vol) for 2 hours at room temperature and incubated with primary antibodies corresponding to DARPP-32, ERK1/2, $\Delta$ fosB, or tau (1:500; Transduction Laboratories, Lexington, $\mathrm{KY}$ ) at $4^{\circ} \mathrm{C}$ overnight. The membranes were subsequently washed with TBST (50 $\mathrm{mM}$ tris(hydroxymethyl)aminomethane hydrochloride [pH 7.5], $150 \mathrm{mM} \mathrm{NaCl}, 0.05 \%$ Tween $^{\circledR} 20$ [Sigma-Aldrich]) and incubated with secondary horseradish peroxidase-conjugated immunoglobulin $\mathrm{G}$ for 1 hour at room temperature. Immunoreactive proteins were visualized by LumiGLO (Cell Signaling Technology, Beverly, MA) chemiluminescent reagent and peroxide. The light-emitting bands were detected with X-ray films.

\section{Immunohistochemistry}

Rats were sacrificed by deep anesthesia using $3 \%$ phenobarbital and their ascending aortas were perfused with cold phosphatebuffered saline (PBS), followed by $4 \%$ paraformaldehyde. Brains were removed and post-fixed in the same fixative for 24 hours, followed by $30 \%$ sucrose. Six series of coronal sections were cut at a thickness of $30 \mu \mathrm{m}$ using a freezing microtome (Leica 1900, Leica Microsystems GmbH, Wetzlar, Germany) and stored at $-20^{\circ} \mathrm{C}$ until used. After endogenous peroxidase was quenched with $3 \%$ hydrogen peroxide, brain sections were preincubated in $10 \%$ normal horse serum $/ 0.2 \%$ Triton X-100/0.1 M PBS for 1 hour at room temperature. The sections were incubated with antibodies against tau and p-tau (1:500; Abcam, Cambridge, MA) in 0.1 M PBS containing $1 \%$ normal horse serum. The sections were then incubated for 1 hour at room temperature with biotinylated anti-mouse immunoglobulin G (1:200; Vector Labs, Burlingame, CA). The bound antibodies were visualized using an avidin-biotinperoxidase complex system (Vectastain ABC Elite Kit, Vector Labs), with 3,39-diaminobenzidine as the chromogen.

\section{Enzyme-linked immunosorbent assay (ELISA)}

Striatal supernatants of rats were measured for dynorphin concentrations using a sandwich ELISA procedure according to the manufacturer's instructions (Cusabio Biotech Co, Ltd, Wuhan, People's Republic of China). Values were converted to $\mathrm{pg} / \mathrm{mL}$ with reference to a standard curve that was assayed in parallel to the test samples. The range of values detected by this assay for dynorphin was $0.32-20 \mathrm{ng} / \mathrm{mL}$.

\section{Statistics}

Data were expressed as the mean \pm standard deviation. Behavioral analyses were performed using a $t$-test. Other statistical analyses were performed using one-way analysis of variance, followed by Dunnett's $t$-test. $P$ values $<0.05$ were considered statistically significant differences.

\section{Results}

\section{Nanoparticle morphology}

The mean particle size of the nanoparticles was approximately $500 \mathrm{~nm}$ and the surface was smooth, with no evident drug particles embedded, which helped to reduce the initial burst. After freeze-drying, the nanoparticles turned into welldispersed powder (Figure 1).

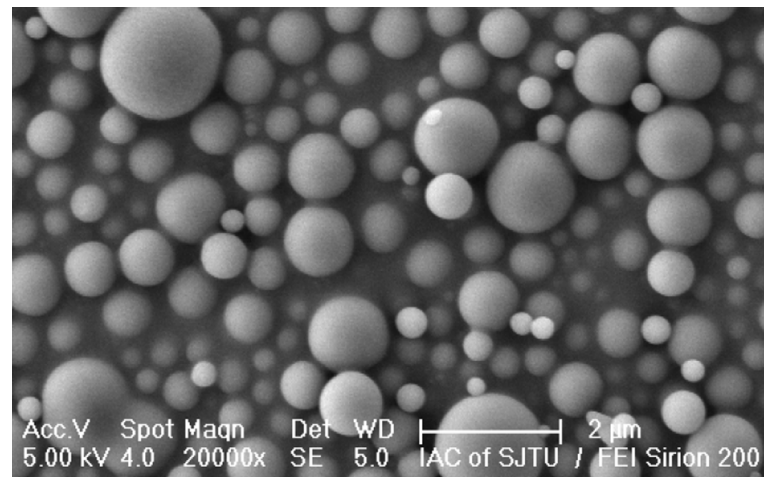

Figure I Scanning electron microscopy of the nanoparticles. 


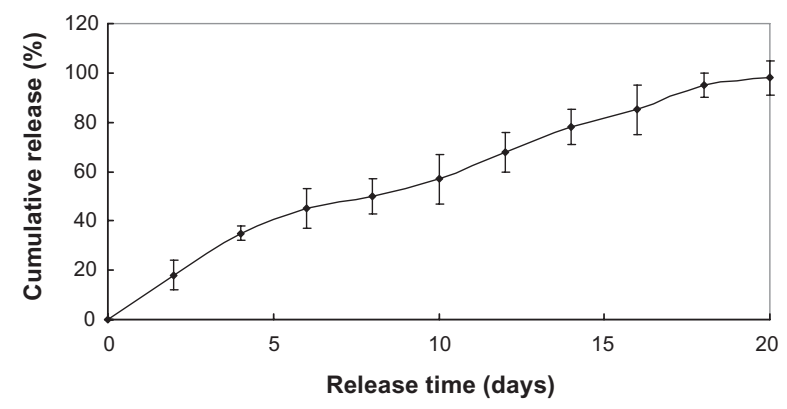

Figure $\mathbf{2}$ Levodopa methyl ester release in vitro.

\section{In vitro release}

Figures 2 and 3 show the two drugs sustained release within approximately 3 weeks, with the initial burst rate only $18.3 \%$ and $30 \%$ total drug loading in the first 2 days. The two drugloaded nanoparticles were preferred to continue the experiment in vivo due to its more stable release.

\section{Encapsulation efficiency}

Encapsulation efficiency was calculated using Equation 1. The encapsulation efficiency of LDME was $60.15 \% \pm 4.2 \%$, lower than that of benserazide $(62.87 \% \pm 6.9 \%)$. Encapsulation efficiency is mainly affected by the way the nanoparticles are prepared; for example, the spray drying process always produces a much higher encapsulation rate. In our study, encapsulation efficiency was influenced by various parameters, such as $\mathrm{pH}$ value when the nanoparticles were formed by fast stirring and the pressure when the organic phase volatilized.

\section{In vivo release}

Plasma concentration of LDME reached a high stable level in the first 14 days then reverted to a relatively normal level until nearly 3 weeks had passed (Figure 4).

The release profile of benserazide was similar to that of LDME, since they share the same release mechanism in vivo (Figure 5).

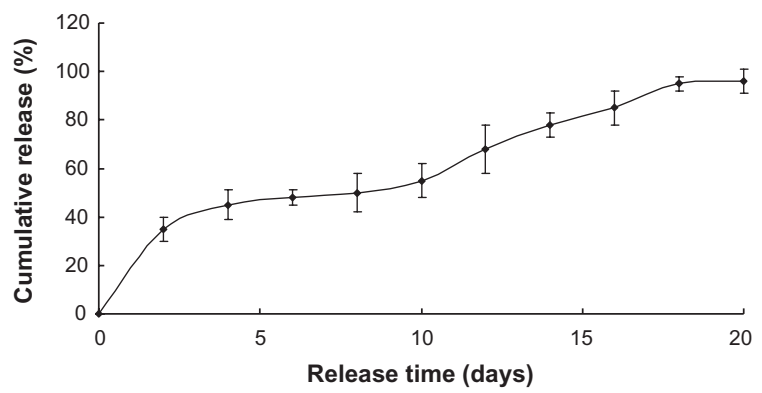

Figure 3 Benserazide release in vitro.

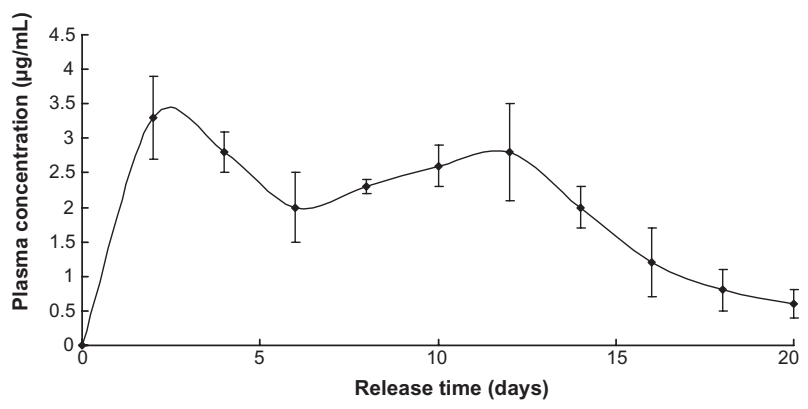

Figure 4 Levodopa methyl ester release in vivo. Note: Nanoparticles injected $=$ I g.

Both LDME and benserazide showed longer sustained release in vivo than in in vitro, probably because, in in vitro, the dissolution medium was refreshed frequently, which provided sink conditions that accelerated the diffusion driven by concentration difference, while the drugs in vivo tended to accumulate at the nanoparticles' position much longer.

\section{Apomorphine-induced rotations}

As shown in Figure 6, after apomorphine administration, apomorphine-induced rotations in rats decreased following administration of levodopa plus benserazide (ie, in the levodopa + levodopa group) or administration of LDME/ benserazide-loaded nanoparticles (ie, in the levodopa + nanoparticle group). More importantly, the degree of reduction of apomorphine-induced rotations was comparable in the two groups. The data showed that the degree of alleviation of parkinsonism in the two groups was comparable.

\section{Effect of administration of LDME/ benserazide-loaded nanoparticles on AIMs in dyskinetic rats}

After administration of levodopa, dyskinetic rats showed increased AIMs. This increase indicated that levodopa can increase the symptoms of dyskinetic rats. However, administration of LDME/benserazide-loaded nanoparticles

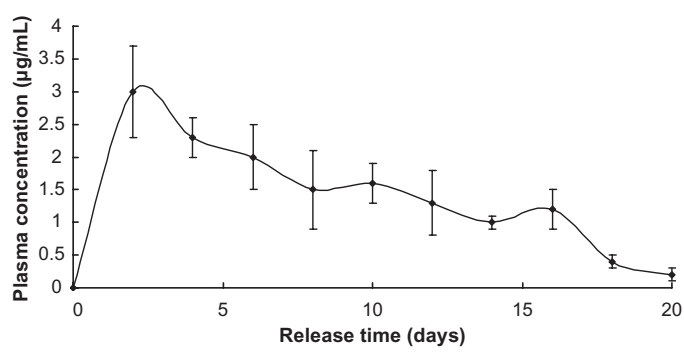

Figure 5 Benserazide release in vivo. Note: Nanoparticles injected $=$ I g. 


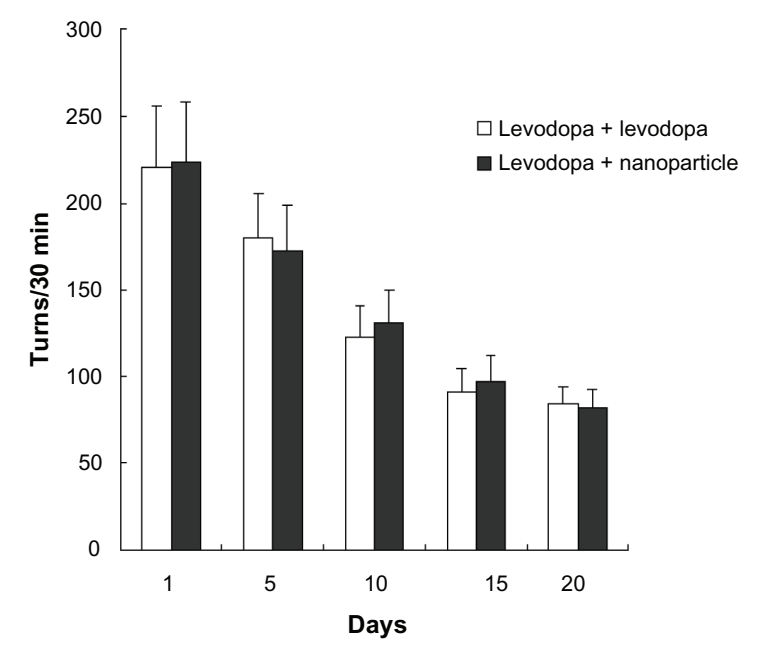

Figure 6 Effect of administration of levodopa methyl ester/benserazide-loaded nanoparticles on apomorphine-induced rotations in dyskinetic rats.

Notes: $\mathrm{n}=12$ per group. Statistical analysis was performed using a $t$-test.

markedly reduced AIMs produced by levodopa/benserazide in dyskinetic rats that had undergone 3-week long dyskinesia priming (Figure 7).

\section{Effect of administration of LDME/ benserazide-loaded nanoparticles on phosphorylated levels of DARPP-32 and $E R K I / 2$ and $\triangle$ fosB in dyskinetic rats}

As shown in Figure 8A and B, administration of LDME plus benserazide significantly increased the levels of p-DARPP-32 and $\mathrm{p}$-ERK $1 / 2$ in dyskinetic rats. In contrast, administration of LDME/benserazide-loaded nanoparticles reduced the levels of p-DARPP-32 and p-ERK1/2 in dyskinetic rats. No significance difference in total DARPP-32 and ERK1/2 was observed in dyskinetic rats treated with LDME plus benserazide or LDME/benserazide-loaded nanoparticles. $\Delta$ fosB levels were determined by Western blot. Upon analysis, we found that administration of LDME plus benserazide induced increased levels of $\Delta$ fosB in dyskinetic rats. However, administration of LDME/benserazide-loaded nanoparticles prevented the increase of $\Delta$ fosB in dyskinetic rats (Figure 8C).

\section{Effect of administration of LDME/ benserazide-loaded nanoparticles on phosphorylated levels of tau protein in dyskinetic rats}

As shown in Figure 9B, administration of LDME/ benserazide-loaded nanoparticles led to a similar pattern of changes in p-tau compared with p-DARPP-32, p-ERK1/2,
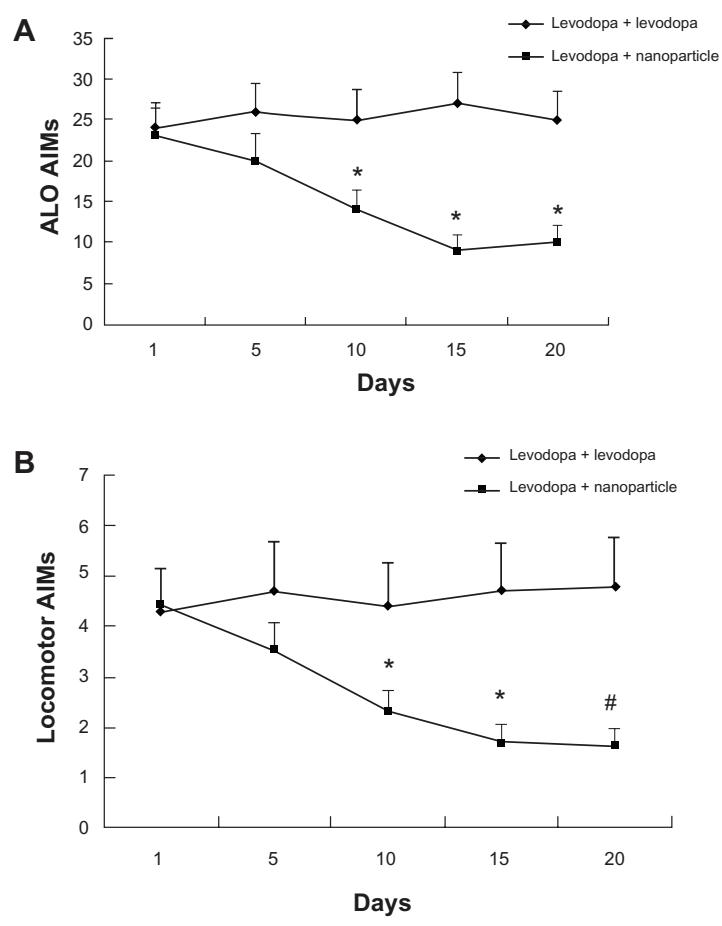

Figure 7 Effect of administration of levodopa methyl ester (LDME)/benserazideloaded nanoparticles on (A) axial, limb, and orolingual (ALO) and (B) locomotive abnormal involuntary movements (AIMs) in dyskinetic rats. After levodopa priming, dyskinetic rats showed increased AIMs. Administration of LDME/benserazide-loaded nanoparticles prevented the increase in dyskinetic rats.

Notes: $\mathrm{n}=12$ per group. Statistical analysis was performed by $t$-test. (A) $* P<0.05$ versus levodopa + levodopa group. (B) $* P<0.05$; $P<0.0$ l versus levodopa + levodopa group.

and $\Delta$ fosB in dyskinetic rats. In general, levodopa treatment produced increased levels of p-tau in dyskinetic rats treated with levodopa when compared with 6-OHDAlesioned rats treated with the vehicle. However, no obvious increase of p-tau was seen in LDME/benserazide-loaded nanoparticle-treated dyskinetic rats compared with vehicle-treated 6-OHDA-lesioned rats. Immunohistochemistry confirmed the results measured by Western blot (Figure 9A).

\section{Effect of administration of LDME/ benserazide-loaded nanoparticles on dynorphin levels of in dyskinetic rats}

The levels of dynorphin in the striatum and cortex of rats were analyzed by ELISA. As shown in Figure 10, dynorphin levels were reduced in the striatum and cortex of PD rats. After repeated administration of LDME plus benserazide, dynorphin levels were increased in the striatum and cortex of dyskinetic rats compared with vehicle-treated 6-OHDA-lesioned rats. In contrast, administration of LDME/ benserazide-loaded nanoparticles prevented the increase of dynorphin in dyskinetic rats. 


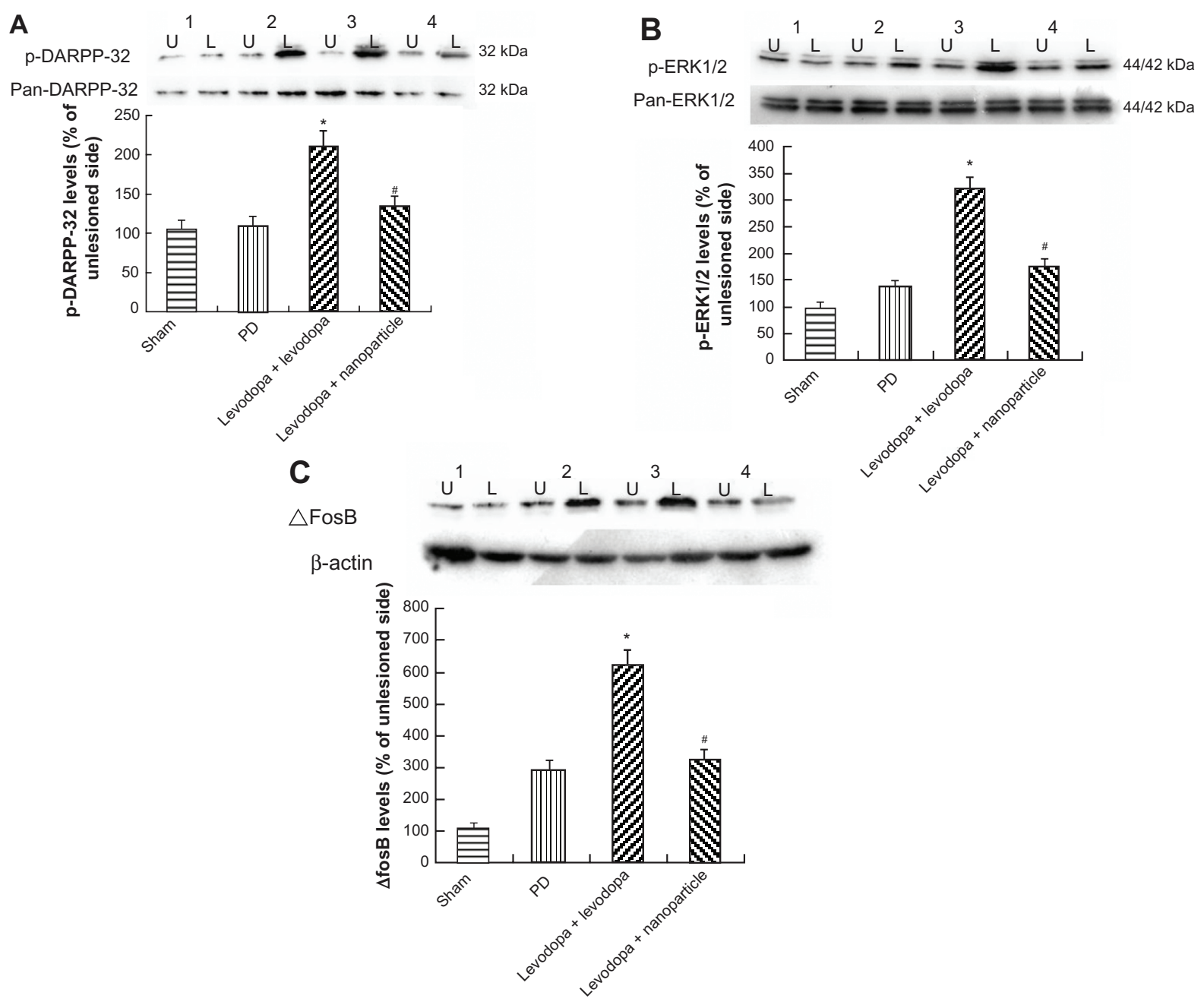

Figure 8 Effect of administration of LDME/benserazide-loaded nanoparticles on levels of p-DARPP-32, p-ERKI/2, and $\Delta$ fosB. Protein levels were determined by Western blot using proteins extracted from the striatum of the rat brain. The results indicate that administration of LDME plus benserazide induced increased levels of p-DARPP-32 (A), P-ERKI/2 (B), and $\triangle$ fosB $(\mathbf{C})$ in dyskinetic rats. Reduced levels of these molecules were found in - dyskinetic rats treated with LDME/benserazide-loaded nanoparticles. Notes: $(\mathbf{A}) * P<0.01$ versus PD group; ${ }^{*} P<0.01$ versus Levodopa + levodopa group. $(\mathbf{B}) * P<0.05$ versus $P D$ group; $\# P<0.0$ I versus Levodopa + levodopa group. $(\mathbf{C}) * P<0.0$ I versus PD group; ${ }^{P} P<0.01$ versus Levodopa + levodopa group. $n=6$ per group. Statistical analysis was performed using one-way analysis of variance, followed by Dunnett's $t$-test. Abbreviations: L, lesioned side; LDME, levodopa methyl ester; PD, Parkinson's disease; U, unlesioned side.

\section{Discussion}

Levodopa-induced dyskinesia is the main side effect of levodopa in the treatment of Parkinson's disease. It has been demonstrated that continuous dopamine stimulation and long-term levodopa are useful in the treatment of LID. ${ }^{11,12}$ However, the mechanism underlying this is not understood. In this study, LID was induced in a rat model of PD by repeated administration of levodopa. We found that ALO AIMs gradually developed during chronic treatment with levodopa. Then LDME/benserazide-loaded nanoparticles, which can release levodopa and benserazide in a sustained manner, were used to treat rats with LID. We found that LDME plus benserazide-treated dyskinetic rats showed sustained AIM scores. However, administration of LDME/ benserazide-loaded nanoparticles can ameliorate LID in established LID rats, which was evidenced by decreased AIMs observed in LID rats. More importantly, molecular makers of LID were reduced in dyskinetic rats. This indicates that LDME/benserazide-loaded nanoparticles can be used to reduce the expression of LID in rats.

Phosphorylation is indicative of ERK1/2 activation, which triggers a molecular cascade that frequently results in immediate early gene expression. ${ }^{13-15}$ The increases in ERK1/2 activation and $\Delta$ fosB expression after chronic treatment appear to be specific to the supersensitive response to levodopa induced by the dopaminergic lesion because neither acute nor chronic levodopa treatment activates ERK1/2 phosphorylation or increases $\Delta$ fosB expression in the dopamine-intact striatum. ${ }^{16,17}$ Phosphorylation and activation of ERK1/2 and subsequent translocation to 
A


B

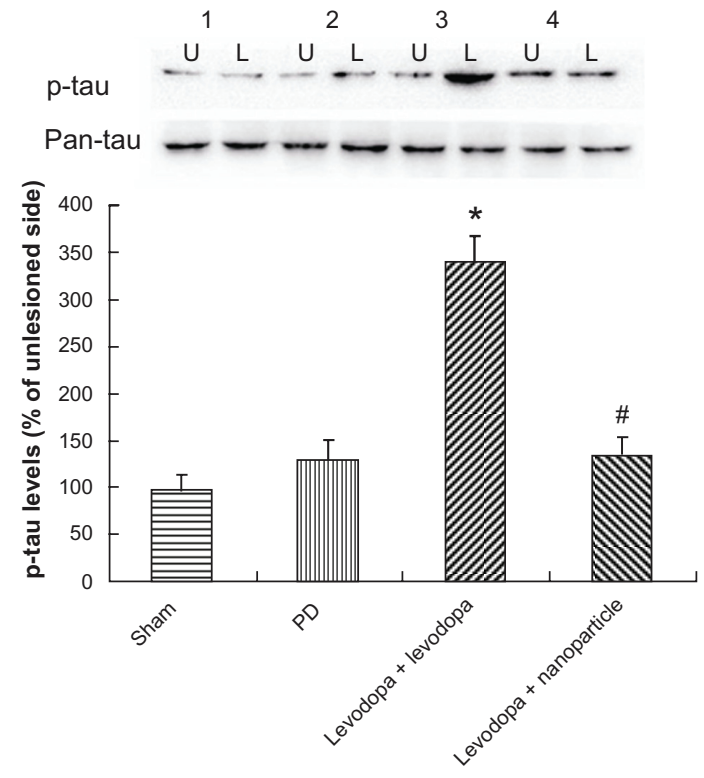

Figure 9 Effect of administration of LDME/benserazide-loaded nanoparticles on p-tau levels. (A) Immunohischemistry results showed that elevated levels of p-tau were found in LDME-plus benserazide-treated dyskinetic rats. Administration of LDME/benserazide-loaded nanoparticles reduced the increase of p-tau in dyskinetic rats. (B) Western blot results showed that administration of LDME plus benserazide induced increased levels of $p$-tau. Administration of LDME/benserazide-loaded nanoparticles prevented the increase of $\mathrm{p}$-tau.

Notes: $(\mathbf{A}) * P<0.01$ versus $P D$ group; ${ }^{*} P<0.0$ I versus levodopa + levodopa. (B) $* P<0.05$ versus PD group; ${ }^{\sharp} P<0.05$ versus levodopa + levodopa. $n=6$ per group. Statistical analysis was performed using one-way analysis of variance, followed by Dunnett's $t$-test. Abbreviations: L, lesioned side; LDME, levodopa methyl ester; PD, Parkinson's disease; $\mathrm{p}$-tau, phosphorylated tau; $\mathrm{U}$, unlesioned side.

the nucleus has been shown to be involved in synaptic plasticity leading to long-term potentiation. ${ }^{18}$ Similarly, phosphorylation of ERK $1 / 2$ and the subsequent long-lasting overexpression of $\Delta$ fosB after levodopa treatment may mediate long-term potentiation at glutamatergic synapses
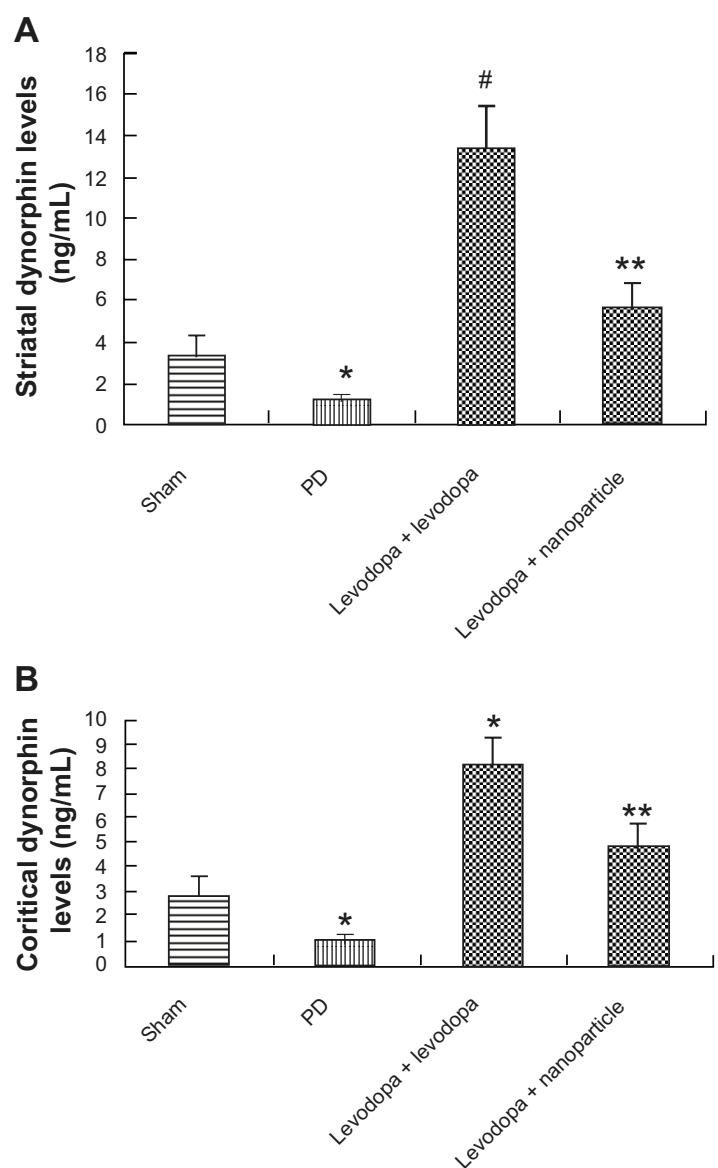

Figure 10 Effect of administration of LDME/benserazide-loaded nanoparticles on dynorphin levels in the striatum (A) and cortex (B) of dyskinetic rats. The levels of dynorphin were evaluated by ELISA. The results showed that after repeated administration of LDME plus benserazide, dynorphin increased significantly in dyskinetic rats. However, administration of LDME/benserazide-loaded nanoparticles decreased dynorphin levels in dyskinetic rats.

Notes: $(\mathbf{A}) * P<0.01$ versus sham; ${ }^{*} P<0.01$ versus $P D$ group; ${ }^{* *} P<0.01$ versus levodopa + levodopa group. (B) $* P<0.05$ versus sham; $\# P<0.0$ I versus $P D$ group; $* p<0.0$ I versus levodopa + nanoparticle group. $\mathrm{n}=6$ per group. Statistical analysis was performed using one-way analysis of variance, followed by Dunnett's $t$-test. Abbreviation: PD, Parkinson's disease.

in the striatum..$^{16,19}$ It has been suggested that hyperactivity in the glutamatergic corticostriatal pathway is involved in the development or expression of dyskinesias. ${ }^{20-22}$ DARPP-32 is known to play a critical role in D1R-mediated transmission. ${ }^{23-25}$ Studies have shown that DARPP-32 is one important mediator of cAMP signaling. PKA-catalyzed phosphorylation at threonine 34 leads DARPP-32 to be an inhibitor of protein phosphatase- $1 .{ }^{26}$ In turn, this prevents dephosphorylation of downstream target proteins regulated by PKA, thereby intensifying cAMP-mediated responses. In this study, we found that chronic levodopa treatment induced increased levels of p-DARPP-32, p-ERK1/2 and $\Delta$ fosB. Meanwhile, levodopa-treated dyskinetic rats showed high AIM scores. This indicated that hyperactivity of neurons in the striata of PD rats can be induced by 
chronic levodopa treatment. In contrast, administration of LDME/benserazide-loaded nanoparticles reduced levels of p-DARPP-32, p-ERK1/2, and $\Delta$ fosB. This indicates that administration of LDME/benserazide-loaded nanoparticles can reduce the hyperactivity of neuron in striata of LID rats. Previous studies have shown that long-term administration of levodopa can lead to the phosphorylation of tau in 6-OHDA lesioned rats. ${ }^{8}$ This indicates that p-tau may play a role in the expression of LID. In this study, we found that after repeated administration of levodopa. Increased levels of p-tau were observed in dyskinetic rats, which was consistent with our previous study. ${ }^{8}$ However, administration of LDME/benserazide-loaded nanoparticles prevented the increase of $\mathrm{p}$-tau in dyskinetic rats while reducing the development of dyskinesia. This suggests that preventing the phosphorylation of tau might be helpful in reducing the emergence of LID in rats.

An association between LID and an upregulation of striatal preprodynorphin mRNA in 6-OHDA-lesioned rats has been widely described. ${ }^{27}$ Reducing the overexpression of dynorphin can attenuate the expression of LID. In animals, some agents have been proven to attenuate the expression of LID by reducing dynorphin levels. ${ }^{28}$ In this study, we found that levodopa induced increased levels of dynorphin in the striatum and cortex of dyskinetic rats, which confirmed the previous study. However, administration of LDME/benserazide-loaded nanoparticles reduced the development of LID in rats by preventing the increase of dynorphin induced by levodopa.

\section{Conclusion}

We found that levodopa treatment can cause the expression of LID in rats. With the expression of dyskinesia, increased levels of p-DARPP-32, p-ERK1/2, $\Delta$ fosB, tau, and dynorphin were seen in dyskinetic rats. However, administration of LDME/benserazide-loaded nanoparticles reduced the expression of LID while preventing the increase of molecules that are closely associated with the development of dyskinesia in dyskinetic rats.

\section{Acknowledgments}

The study was supported by the Special Nano Project of the Shanghai Committee of Science and Technology, China (11nm0503300 and 1052nm03900), Projects of the Shanghai Committee of Science and Technology, People's Republic of China (0952nm03700 and 11410708900), Program of Shanghai Municipal Education Commission, People's Republic of China (10ZZ72), National Science Foundation of China Committee (81071025 and 81171203), and $\mathrm{PhD}$ Programs Foundation of Ministry of Education of China (20090073120085). We also appreciate the generous help from Faculty of Instrumental Analysis Centre (IAC) of Shanghai Jiaotong University.

\section{Disclosure}

The authors declare no competing financial interests or other conflicts of interest in this work.

\section{References}

1. Eskow KL, Dupre KB, Barnum CJ, Dickinson SO, Park JY, Bishop C. The role of the dorsal raphe nucleus in the development, expression, and treatment of L-dopa-induced dyskinesia in hemiparkinsonian rats. Synapse. 2009;63(7):610-620.

2. Jaunarajs KL, Dupre KB, Steiniger A, et al. Serotonin 1B receptor stimulation reduces D1 receptor agonist-induced dyskinesia. Neuroreport. 2009;20(14):1265-1269.

3. Carta M, Carlsson T, Kirik D, Björklund A. Dopamine released from 5-HT terminals is the cause of L-DOPA-induced dyskinesia in parkinsonian rats. Brain. 2007;130(Pt 7):1819-1833.

4. Jenner P. Preventing and controlling dyskinesia in Parkinson's disease a view of current knowledge and future opportunities. Mov Disord. 2008;23(Suppl 3):S585-S598.

5. Björklund T, Carlsson T, Cederfjäll EA, Carta M, Kirik D. Optimized adeno-associated viral vector-mediated striatal DOPA delivery restores sensorimotor function and prevents dyskinesias in a model of advanced Parkinson's disease. Brain. 2010;133(Pt 2):496-511.

6. Stocchi F. The therapeutic concept of continuous dopaminergic stimulation (CDS) in the treatment of Parkinson's disease. Parkinsonism Relat Disord. 2009;15(Suppl 3):S68-S71.

7. Ren T, Yang X, Wu N, Cai Y, Liu Z, Yuan W. Sustained-release formulation of levodopa methyl ester/benserazide for prolonged suppressing dyskinesia expression in 6-OHDA-leisoned rats. Neurosci Lett. 2011;502(2):117-122.

8. Lebel M, Chagniel L, Bureau G, Cyr M. Striatal inhibition of PKA prevents levodopa-induced behavioural and molecular changes in the hemiparkinsonian rat. Neurobiol Dis. 2010;38(1):59-67.

9. Paxinos G, Watson C. The Rat Brain in Stereotaxic Coordinates, 6th ed Sydney, Australia: Academic Press; 2007.

10. Morgese MG, Cassano T, Cuomo V, Giuffrida A. Anti-dyskinetic effects of cannabinoids in a rat model of Parkinson's disease: role of CB(1) and TRPV1 receptors. Exp Neurol. 2007;208(1):110-119.

11. Kim DS, Palmiter RD, Cummins A, Gerfen CR. Reversal of supersensitive striatal dopamine D1 receptor signaling and extracellular signalregulated kinase activity in dopamine-deficient mice. Neuroscience. 2006;137(4):1381-1388.

12. Smith LA, Jackson MJ, Al-Barghouthy G, et al. Multiple small doses of levodopa plus entacapone produce continuous dopaminergic stimulation and reduce dyskinesia induction in MPTP-treated drug-naive primates. Mov Disord. 2005;20(3):306-314.

13. Lindgren HS, Ohlin KE, Cenci MA. Differential involvement of D1 and D2 dopamine receptors in L-DOPA-induced angiogenic activity in a rat model of Parkinson's disease. Neuropsychopharmacology. 2009;34(12):2477-2488.

14. Santini E, Valjent E, Usiello A, et al. Critical involvement of cAMP/ DARPP-32 and extracellular signal-regulated protein kinase signaling in L-DOPA-induced dyskinesia. J Neurosci. 2007;27(26): 6995-7005.

15. Santini E, Alcacer C, Cacciatore S, et al. L-DOPA activates ERK signaling and phosphorylates histone $\mathrm{H} 3$ in the striatonigral medium spiny neurons of hemiparkinsonian mice. J Neurochem. 2009;108(3): $621-633$. 
16. Pavón N, Martín AB, Mendialdua A, Moratalla R. ERK phosphorylation and FosB expression are associated with L-DOPA-induced dyskinesia in hemiparkinsonian mice. Biol Psychiatry. 2006;59(1):64-74.

17. Westin JE, Vercammen L, Strome EM, Konradi C, Cenci MA. Spatiotemporal pattern of striatal ERK1/2 phosphorylation in a rat model of L-DOPA-induced dyskinesia and the role of dopamine D1 receptors. Biol Psychiatry. 2007;62(7):800-810.

18. Iravani MM, Jenner P. Mechanisms underlying the onset and expression of levodopa-induced dyskinesia and their pharmacological manipulation. J Neural Transm. 2011;118(12):1661-1690.

19. Ravenscroft P, Chalon S, Brotchie JM, Crossman AR. Ropinirole versus L-DOPA effects on striatal opioid peptide precursors in a rodent model of Parkinson's disease: implications for dyskinesia. Exp Neurol. 2004;185(1):36-46.

20. Marin C, Bonastre M, Aguilar E, Jiménez A. The metabotropic glutamate receptor antagonist 2-methyl-6-(phenylethynyl) pyridine decreases striatal VGlut 2 expression in association with an attenuation of L-dopainduced dyskinesias. Synapse. 2011;65(10):1080-1086.

21. Levandis G, Bazzini E, Armentero MT, Nappi G, Blandini F. Systemic administration of an mGluR5 antagonist, but not unilateral subthalamic lesion, counteracts 1-DOPA-induced dyskinesias in a rodent model of Parkinson's disease. Neurobiol Dis. 2008;29(1):161-168.

22. Yamamoto N, Soghomonian JJ. Metabotropic glutamate mGluR5 receptor blockade opposes abnormal involuntary movements and the increases in glutamic acid decarboxylase mRNA levels induced by 1-DOPA in striatal neurons of 6-hydroxydopamine-lesioned rats. Neuroscience. 2009;163(4):1171-1180.
23. Greengard P. The neurobiology of slow synaptic transmission. Science. 2001;294(5544):1024-1030.

24. Kuan WL, Zhao JW, Barker RA. The role of anxiety in the development of levodopa-induced dyskinesias in an animal model of Parkinson's disease, and the effect of chronic treatment with the selective serotonin reuptake inhibitor citalopram. Psychopharmacology (Berl). 2008;197(2):279-293.

25. Calabresi P, Di Filippo M, Ghiglieri V, Picconi B. Molecular mechanisms underlying levodopa-induced dyskinesia. Mov Disord. 2008;23(Suppl 3):S570-S579.

26. Aubert I, Guigoni C, Håkansson K, et al. Increased D1 dopamine receptor signaling in levodopa-induced dyskinesia. Ann Neurol. 2005;57(1):17-26.

27. Bové J, Serrats J, Mengod G, Cortés R, Aguilar E, Marin C. Reversion of levodopa-induced motor fluctuations by the A2A antagonist CSC is associated with an increase in striatal preprodynorphin mRNA expression in 6-OHDA-lesioned rats. Synapse. 2006;59(7):435-444.

28. Tamim MK, Samadi P, Morissette M, et al. Effect of non-dopaminergic drug treatment on Levodopa induced dyskinesias in MPTP monkeys: common implication of striatal neuropeptides. Neuropharmacology. 2010;58(1):286-296.
International Journal of Nanomedicine

\section{Publish your work in this journal}

The International Journal of Nanomedicine is an international, peerreviewed journal focusing on the application of nanotechnology in diagnostics, therapeutics, and drug delivery systems throughout the biomedical field. This journal is indexed on PubMed Central, MedLine, CAS, SciSearch ${ }^{\circledR}$, Current Contents ${ }^{\circledR} /$ Clinical Medicine,

\section{Dovepress}

Journal Citation Reports/Science Edition, EMBase, Scopus and the Elsevier Bibliographic databases. The manuscript management system is completely online and includes a very quick and fair peer-review system, which is all easy to use. Visit http://www.dovepress.com/ testimonials.php to read real quotes from published authors. 